\title{
O descortinar de uma epigastralgia
}

Ana Correia de Oliveira, ${ }^{1}$ Rosário Mendonça e Moura, ${ }^{1}$ Paulo Pessanha ${ }^{2}$

\section{RESUMO}

Introdução: A dor epigástrica está frequentemente associada à patologia digestiva. Contudo, é essencial a realização de uma anamnese e exame objetivo cuidadosos, pois a queixa referida também pode estar associada a outras patologias.

Descrição do Caso: Homem de 41 anos, com os seguintes problemas de saúde ativos: tabagismo, dislipidemia, hipertensão arterial e excesso de peso. Antecedentes familiares relevantes de doença coronária com morte precoce (pai faleceu aos 46 anos por enfarte agudo do miocárdio).

Em julho de 2014 recorre a clínica privada por dor epigástrica súbita. Foram realizadas analgesia e proteção gástrica e o doente apresentou melhoria clínica. De acordo com o mesmo, a anamnese e o exame objetivo foram breves. Após um mês teve novo episódio idêntico, não tendo recorrido a avaliação médica. Em setembro de 2014 recorre à Unidade de Saúde Familiar (USF) para solicitar exames digestivos (endoscopia digestiva alta e colonoscopia). Na consulta refere que, durante os episódios de dor epigástrica, experienciou uma sensação de dormência do membro superior esquerdo. Foram requisitados exames complementares, nomeadamente estudo analítico, ecografia abdominal, eletrocardiograma (ECG) e prova de esforço. Após realização de exames complementares foi encaminhado para consulta de cardiologia de urgência e realizada cirurgia de revascularização coronária por doença de três vasos.

Comentário: A incidência de patologia cardíaca está a aumentar, sendo os fatores de risco cardiovascular cumulativos para o aparecimento de doença cardiovascular. Este doente apresentava inúmeros fatores de risco que não poderiam ser negligenciados, devendo ter sido considerada a hipótese de uma dor isquémica atípica desde o início. Concluindo, o médico deve conhecer os motivos mais prevalentes associados aos sintomas referidos pelo doente. Contudo, não deve excluir apresentações atípicas ou diagnósticos diferenciais menos frequentes face às queixas apresentadas.

Palavras-chave: Dor abdominal; Enfarte agudo do miocárdio; Relato de caso.

\section{INTRODUÇÃO}

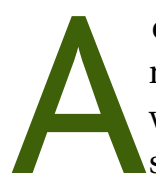
doença cardiovascular (DCV), nomeadamente a doença coronária (DC) e o acidente vascular cerebral (AVC), são a principal causa de morte prematura a nível mundial. ${ }^{1}$ Apesar da mortalidade por doença cardiovascular estar a diminuir na maioria dos países desenvolvidos, sabe-se que a sua prevalência está a aumentar mundialmente. ${ }^{2}$

O termo enfarte agudo do miocárdio (EAM) é utilizado quando há evidência de necrose miocárdica num contexto clínico consistente com isquemia aguda do miocárdio. Quando tal não acontece, ou seja, quando há evidência de necrose mas não há contexto clínico, designa-se apenas de enfarte do miocárdio. ${ }^{3}$

'Médica interna de Medicina Geral e Familiar, USF São João do Porto ${ }^{2}$ Médico Assistente Graduado de Medicina Geral e Familiar, USF São João do Porto
Tipicamente, o EAM apresenta-se como angina. No entanto, em alguns doentes não há dor pré-cordial mas somente dor ao nível do braço, pescoço, mandíbula ou desconforto epigástrico. ${ }^{4}$

Este caso ilustra o papel do médico de família na abordagem de sintomas inespecíficos, que poderão estar presentes em mais do que uma patologia, nomeadamente cardíaca e digestiva. O médico de família é um elemento chave na prevenção e diagnóstico das DCV.

\section{DESCRIÇÃO DO CASO}

A.C. é um utente do sexo masculino, caucasiano, de 41 anos de idade, casado e com um filho. Trata-se de uma família nuclear na fase IV do ciclo de vida de Duvall. É empregado numa garagem de automóveis. Tem como problemas de saúde tabagismo (20 cigarros/dia, 


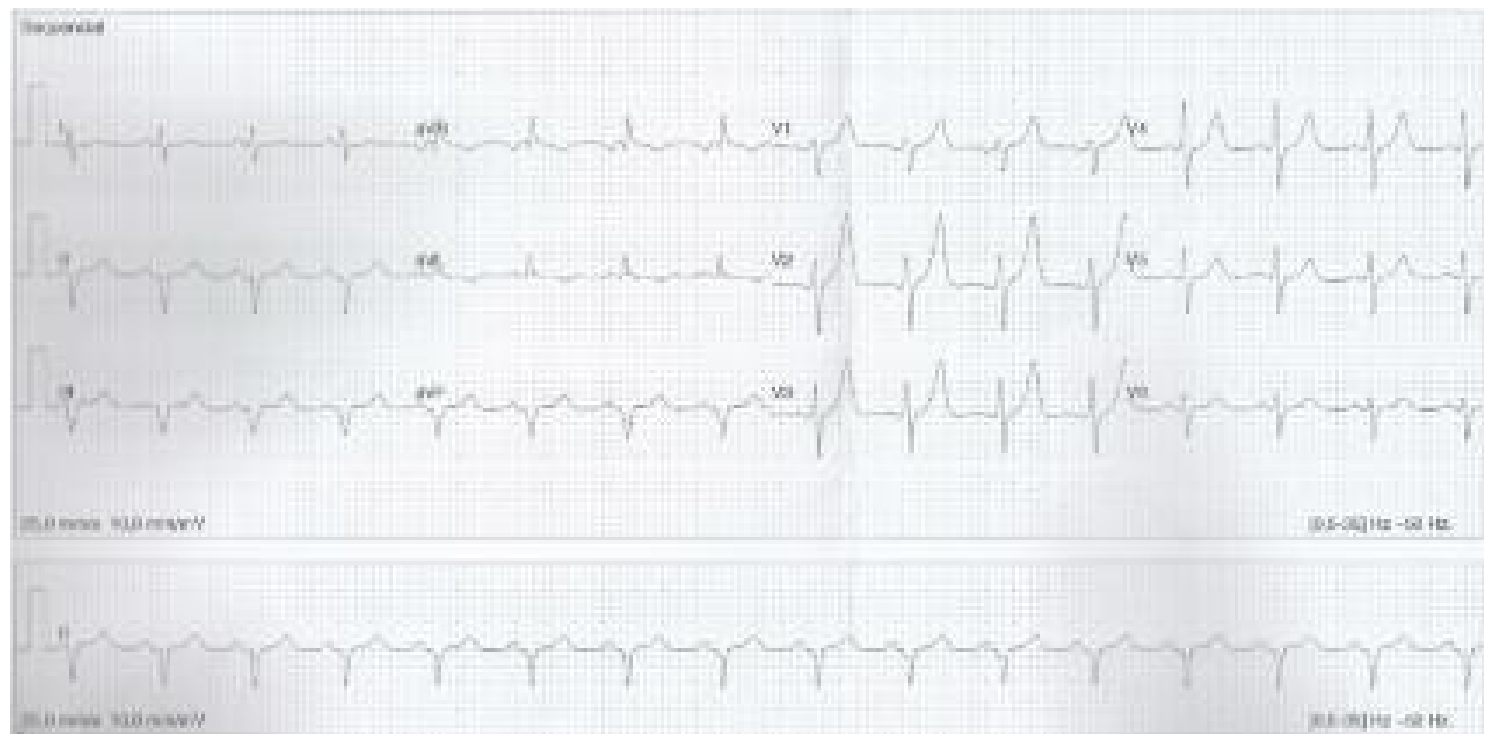

Figura 1. Eletrocardiograma do doente.

20 unidades de maço/ano), dislipidemia (colesterol total 213mg/dL, colesterol HDL 39mg/dL, colesterol LDL $146,2 \mathrm{mg} / \mathrm{dL}$ ), hipertensão arterial (HTA) medicada e controlada (pressão arterial $138 / 81 \mathrm{mmHg}$ ) e excesso de peso (IMC $27,8 \mathrm{~kg} / \mathrm{m}^{2}$ ). Consumo de álcool de $120 \mathrm{~g} /$ semana. O doente nega consumo de substâncias de abuso. A medicação habitual de A.C. era sinvastatina $20 \mathrm{mg}$ id e ramipril $5 \mathrm{mg}$ id.

A história da doença atual inicia-se em julho de 2014, altura em que A.C. recorre ao médico numa clínica privada por dor epigástrica, súbita, de intensidade 3 em 10, sem fatores desencadeantes, de alívio ou agravamento e sem irradiação da mesma. Segundo o doente, foi uma consulta rápida com exame objetivo escasso. Nesta altura foi medicado com protetor gástrico e indicação para analgesia.

Em agosto de 2014 refere repetição dos sintomas, não tendo procurado assistência médica. Em setembro de 2014 recorre então ao seu médico de família para solicitar a realização de endoscopia digestiva alta (EDA) e colonoscopia. Após colheita da anamnese, com descrição de dormência do membro superior esquerdo durante os episódios de dor epigástrica, é colocada a hipótese de patologia cardíaca versus patologia digestiva. Na anamnese é também verificado que o doente possui história familiar de doença coronária com mor- te precoce (pai falecido aos 46 anos por enfarte agudo do miocárdio). Neste momento é explicado ao doente que deve realizar análises, ecografia abdominal e eletrocardiograma (ECG) com prova de esforço e, consoante os resultados, a necessidade de realizar a EDA e colonoscopia. Os exames demonstraram dislipidemia (triglicerídeos 198mg/dL, colesterol total $194 \mathrm{mg} / \mathrm{dL}$, colesterol HDL 40mg/dL, colesterol LDL 114,4mg/dL), aumento da gama-glutamil transferase (GGT) $46 \mathrm{UI} / \mathrm{L}$ e hepatomegalia com infiltração esteatósica. O ECG (Figura 1) mostrou sequelas de necrose inferior. A prova de esforço traduziu prova máxima, sem alterações eletrocardiográficas sugestivas de isquemia do miocárdio, ou seja, foi uma prova negativa perante sintomas.

Após a análise dos exames suspeitou-se que o doente teve dor epigástrica causada por EAM (enfarte agudo do miocárdio) da parede inferior. Foi solicitada a realização de ecocardiograma transtorácico e procedeu-se a alteração da medicação habitual. A sinvastatina foi alterada para rosuvastatina $20 \mathrm{mg}$ id, mantevese ramipril 5mg id e introduziu-se ácido acetilsalicílico $100 \mathrm{mg}$ id, bisoprolol $5 \mathrm{mg}$ id e pantoprazol $40 \mathrm{mg}$ id. Foi ainda efetuada educação para a saúde com controlo dos fatores de risco, nomeadamente evicção tabágica (atualmente 5 cigarros/dia), evicção alcoólica e exercício físico leve a moderado. O ecocardiograma, realiza- 


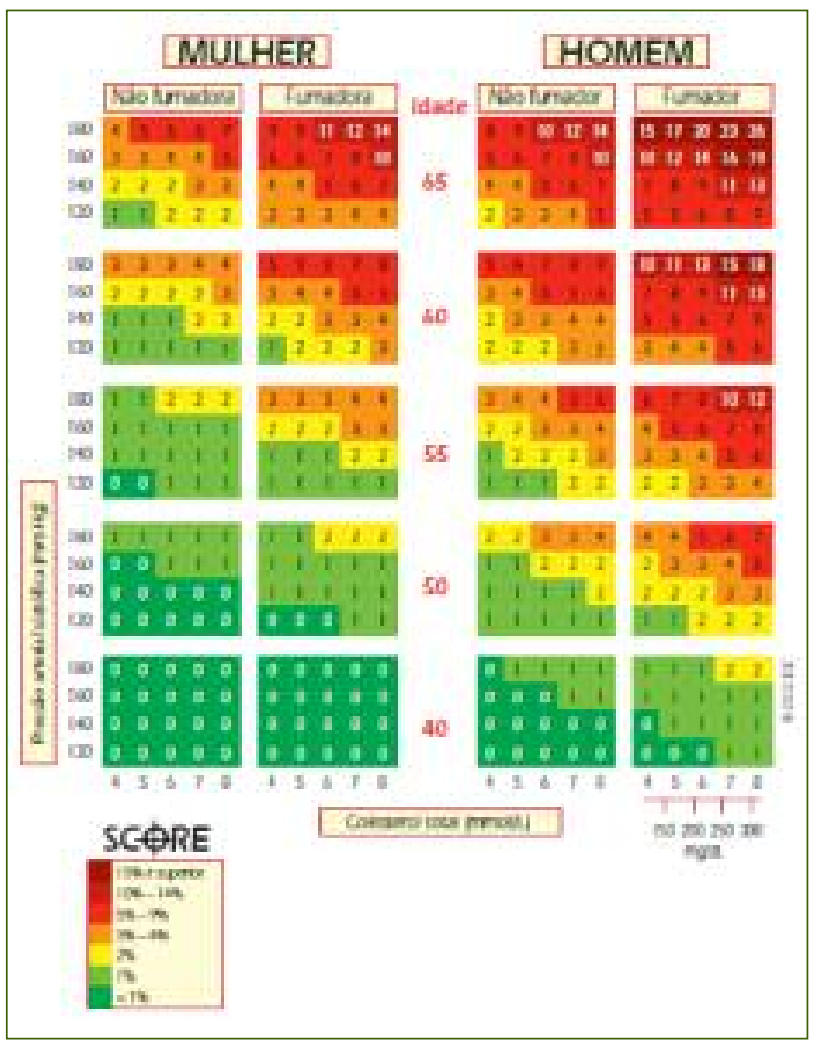

Figura 2. Risco a 10 anos de DCV fatal em regiões da Europa de baixo risco. ${ }^{8}$

do em setembro de 2014, mostrou "ventrículo esquerdo ligeiramente dilatado com alterações da motilidade segmentar: acinésia dos segmentos basais da parede inferior e septo-inferior e apical do septo; hipocinésia de todos os restantes segmentos e paredes. Função ventricular sistólica esquerda moderadamente deprimida".

De acordo com estes antecedentes familiares e resultados de exames é proposto ao doente ser enviado a consulta urgente de cardiologia, que o doente aceita. A coronariografia revelou doença grave de três vasos, com estenose de $70 \%$ do segmento proximal da artéria descendente anterior, artéria circunflexa com oclusão crónica proximal e artéria coronária direita com estenoses suboclusivas, tendo sido encaminhado para a consulta de cirurgia cardiotorácica. Tendo a cintigrafia de perfusão miocárdica revelado viabilidade e isquemia da parede anterior e septo e o doente apresentar angor de esforço típico classe III pouco frequente, nunca em repouso, foi proposto para realização de cirurgia de re- vascularização coronária (CABG) da descendente anterior (DA) e descendente posterior (DP). Enquanto aguardava cirurgia teve agravamento de angor e parestesia dos dedos das mãos, sobretudo à direita; por este motivo foi colocado em lista de espera com urgência. Em julho de 2015 foi chamado para CABG de três vasos, sem intercorrências.

O doente teve alta, assintomático, com consulta agendada, mantendo-se em cessação tabágica e iniciou dupla antiagregação, associando-se clopidogrel $75 \mathrm{mg}$.

\section{COMENTÁRIO}

No caso descrito, A.C. apresentou um quadro clínico de dor epigástrica, tendo o médico, numa primeira abordagem, considerado como hipótese diagnóstica patologia digestiva. Perante um doente com vários fatores de risco cardiovasculares, nomeadamente tabagismo, excesso de peso, HTA, dislipidemia, não deveria ter sido descartada a hipótese de patologia cardíaca. É necessário ter em consideração que a anamnese e exame físico adequados são imprescindíveis. Tendo em conta que, atualmente, os médicos são constantemente pressionados a realizar consultas em tempo escasso, pode levar, por vezes, a anamneses inadequadas com diagnósticos erróneos. Neste caso, o doente referiu que, devido à rapidez da primeira consulta, não teve tempo de informar o médico sobre a sensação de dormência no membro superior esquerdo e tal também não foi questionado. Um EAM pode apresentar-se como dor pré-cordial, no membro superior, mandíbula, desconforto epigástrico, equivalente isquémico (dispneia, cansaço) ou ser assintomático (mais frequente no sexo feminino, idosos ou diabéticos). ${ }^{5} \mathrm{~A}$ primeira consulta realizou-se no sistema privado pois, de acordo com o doente, estava de férias e tinha uma clínica médica numa área próxima. No entanto, considera que o atendimento foi rápido e impreciso, com uma anamnese descurada e exame físico quase inexistente. Apesar de não serem conclusivos, estes dois passos da consulta são imprescindíveis para um diagnóstico assertivo. De acordo com o doente, o motivo que o fez recorrer ao médico de família, numa segunda consulta, deveu-se à confiança depositada há muitos anos e, apesar de não recorrer muitas vezes ao seu médico de família, sentiu a necessidade de acompanhamento sustentado de um 


\section{QUADRO I. Outros fatores de risco'}

\section{Estimativa do risco com recurso à tabela SCORE: os qualificadores}

- A tabela deve ser usada à luz do conhecimento e decisão do clínico, especialmente no que diz respeito às condições locais.

- Tal como em qualquer sistema de avaliação do risco, este poderá ser sobrestimado nos países cuja mortalidade por DCV esteja a diminuir e subestimado se a mortalidade estiver a aumentar.

O risco poderá ser mais elevado do que o indicado na tabela em:

- Pessoas sedentárias ou obesas, especialmente com obesidade central;

- Pessoas com familiar directo portador de DCV prematura.

- Pessoas socialmente desfavorecidas

- Pessoas com nível de colesterol HDL baixo ou nível elevado de triglicéridos de triglicéridos.

- Pessoas com diabetes (sem outros factores de risco ou lesão de órgão alvo): o risco poderá ser 5 vezes superior em mulheres e 3 vezes superior em homens comparado com o de indivíduos sem diabetes mellitus.

- Pessoas assintomáticas com evidência de arteriosclerose subclínica, por exemplo um índice baixo tornozelo-braço ou por imagiologia (ultra-sonografia Doppler) carotídea ou topografia computadorizada)

médico que o "olhasse como eu sou" (sic). Assim, a observação pelo médico de família é muito importante, pois só este profissional tem as características necessárias para a observação e orientação cuidadas, cruzando essa informação com a observação longitudinal prévia. Permite, por exemplo, conhecer bem os doentes e descartar mais facilmente alguma sintomatologia psicológica camuflada pela sintomatologia orgânica sentida.

Calculando o risco cardiovascular pela tabela SCORE, previamente ao episódio de EAM, podemos concluir que o score do doente era de $1 \%$. Porque Portugal se encontra numa região da Europa de baixo risco, ${ }^{1}$ estamos perante um indivíduo do sexo masculino, 41 anos de idade, fumador, com valor de colesterol total de $213 \mathrm{mg} / \mathrm{dl}$ e tensão arterial sistólica de $138 \mathrm{mmHg}$ (Figura 2). No entanto, é necessário considerar que o risco poderá ser mais elevado do que o indicado na tabela; no caso deste doente, ele tinha nível de triglicerídeos elevado, colesterol HDL baixo e familiar portador de DVC prematura (Quadro I). ${ }^{1}$ E fundamental ter em consideração que não podemos olhar apenas para as tabelas de risco a 10 anos de DCV fatal; sabe-se que existem outros fatores, como neste caso, que influenciam significativamente o risco e que têm de ser considerados numa boa anamnese. Após a ocorrência do EAM, este doente passa a ser um doente de risco mui- to alto.

Concluindo, é necessário efetuar uma anamnese assertiva e direcionada para a patologia principal que abranja a sintomatologia referida pelo doente. Contudo, o médico deve ponderar outros diagnósticos diferenciais, sobretudo em doentes de risco. No caso de risco cardiovascular, o médico deve estar ciente dos sintomas atípicos e estar alerta para a possibilidade de ocorrência de eventos tromboembólicos, mesmo que os sintomas sejam insipientes. A medicina deve ser sempre baseada no doente e nas suas necessidades. A consulta deve demorar o tempo necessário para um diagnóstico correto, de modo a levar a um tratamento eficaz para o melhor benefício do doente.

\section{REFERÊNCIAS BIBLIOGRÁFICAS}

1. Perk J, Backer GD, Gohlke H, Graham I, Reiner Z, Verschuren WM, et al. Recomendações Europeias para a prevenção da doença cardiovascular na prática clínica (versão de 2012). Rev Port Cardiol. 2013;32(6):e1e77.

2. Brookes L. INTERHEART: a global case-control study of a risk factors for acute myocardial infarction [Internet]. Medscape; 2014 Sep 21 [cited 2015 Jul 25]. Available from: http://www.medscape.com/viewarticle/489738

3. Thygesen K, Alpert JS, Jaffe AS, Simoons ML, Chaitman BR, White HD, et al. Third universal definition of myocardial infarction. Circulation. 2012;126(16):2020-35.

4. Coven DL, Shirani J, Kalyanasundaram A. Acute coronary syndrome clinical presentation [Internet]. Medscape; 2016 [cited 2015 Jul 27; up- 
dated 2015 Sep 9]. Available from: http://emedicine.medscape.com/article/1910735-clinical

5. Thygesen K, Alpert JS, Jaffe AS, Simoons ML, Chaitman BR, White HD, et al. Third universal definition of myocardial infarction. Eur Heart J. 2012;33(20):2551-67.

6. Ray KK, Kastelein JJ, Boekholdt SM, Nicholls SJ, Khaw KT, Ballantyne $\mathrm{CM}$, et al. The ACC/AHA 2013 guideline on the treatment of blood cholesterol to reduce atherosclerotic cardiovascular disease risk in adults: the good the bad and the uncertain: a comparison with ESC/EAS guidelines for the management of dyslipidaemias 2011. Eur Heart J. 2014;35(15):960-8.

7. Direção-Geral da Saúde. Abordagem terapêutica das dislipidemias no adulto: norma n019/2011, de 28/09/2011; atualização de 30/07/2015. Lisboa: DGS; 2015.
8. Direção-Geral da Saúde. Avaliação do risco cardiovascular SCORE (Systematic Coronary Risk Evaluation): norma n 5/3013, de 19/03/2013; atualização de 21/01/2015. Lisboa: DGS; 2015.

\section{CONFLITO DE INTERESSES}

Os autores declaram não ter conflitos de interesses.

\section{ENDEREÇO PARA CORRESPONDÊNCIA}

Ana Margarida Ribeiro Correia de Oliveira

Rua D. António Barroso n $2311^{\circ}$ Dir 4050-060 Porto

Email: correiadra@gmail.com

Recebido em 20-03-2016

Aceite para publicação em 22-09-2016

\section{ABSTRACT}

\section{UNRAVELING EPIGASTRIC PAIN: A CASE REPORT}

Introduction: Epigastric pain is frequently associated with gastrointestinal disease. However, when faced with a patient complaining of epigastric pain it is important to obtain a careful history and perform a physical examination because this may also be associated with other diseases.

Case Description: We present the story of a 41 year-old man with a history of smoking, dyslipidemia, hypertension and obesity. His father died at age 46 from an acute myocardial infarction. In July 2014 he came to a private clinic complaining of epigastric pain. Following a brief history and physical examination, he received analgesia and gastric mucosal protection with improvement in his symptoms. One month later, he experienced a similar episode of pain, without recourse to medical assistance. In September 2014, came to his family doctor and requested further examinations, including gastroscopy and colonoscopy. The patient also reported that during episodes of epigastric pain he had also experienced a sense of left upper limb numbness. The doctor ordered additional tests, including biochemical analyses, an abdominal ultrasound, an electrocardiogram (ECG), and a stress test. The patient was then referred for an urgent consultation with a cardiologist and coronary artery bypass surgery was performed for triple vessel disease.

Discussion: The incidence of heart disease is increasing along with the prevalence of risk factors for cardiovascular disease. This patient had several risk factors that might suggest atypical ischemic pain as the cause of his epigastric pain. Physicians are aware of the most common causes of symptoms reported by the patient but must also consider atypical presentations of less common diagnoses.

Keywords: Abdominal pain; Myocardial infarction; Case report. 